\title{
Corrigendum
}

\section{Corrigendum to "SIAT-WEXv2: A Wearable Exoskeleton for Reducing Lumbar Load during Lifting Tasks"}

\author{
Xinyu Ji $\mathbb{D}^{1,2,3,4}$ Dashuai Wang $\mathbb{D}^{1,2,4}$ Pengfei Li $\mathbb{D}^{1,2,4}$ Liangsheng Zheng ${ }^{1}{ }^{1,2,4}$ \\ Jianquan Sun $D^{1,2,4}$ and Xinyu $W u$ iD ${ }^{1,2,4}$ \\ ${ }^{1}$ Guangdong Provincial Key Lab of Robotics and Intelligent System, Shenzhen Institutes of Advanced Technology, \\ Chinese Academy of Sciences, Shenzhen, China \\ ${ }^{2}$ CAS Key Laboratory of Human-Machine Intelligence-Synergy Systems, Shenzhen Institutes of Advanced Technology, \\ Shenzhen, China \\ ${ }^{3}$ Wuhan University of Technology, Wuhan, China \\ ${ }^{4}$ Guangdong-Hong Kong-Macao Joint Laboratory of Human-Machine Intelligence-Synergy Systems, Shenzhen, China \\ Correspondence should be addressed to Xinyu Wu; xy.wu@siat.ac.cn
}

Received 31 March 2021; Accepted 31 March 2021; Published 17 April 2021

Copyright ( 2021 Xinyu Ji et al. This is an open access article distributed under the Creative Commons Attribution License, which permits unrestricted use, distribution, and reproduction in any medium, provided the original work is properly cited.

In the article titled "SIAT-WEXv2: A Wearable Exoskeleton for Reducing Lumbar Load during Lifting Tasks" [1], there were two errors in the Abstract and Introduction section which were introduced by the authors during the conversion of unit from $\mathrm{kgf}$ to $\mathrm{N}$ in the revision stage. The corrections should be applied as follows:

(1) In the abstract, the value $28 \mathrm{~N}$ should be corrected to $280 \mathrm{~N}$

(2) In the fourth paragraph of the Introduction section, the value $25 \mathrm{~N}$ should be corrected to $250 \mathrm{~N}$

The author apologizes for the mistake, and the editorial board agrees to the publication of a corrigendum.

\section{Conflicts of Interest}

The authors declare that they have no conflicts of interest.

\section{References}

[1] X. Ji, D. Wang, P. Li, L. Zheng, J. Sun, and X. Wu, "SIATWEXv2: a wearable exoskeleton for reducing lumbar load during lifting tasks," Complexity, vol. 2020, Article ID 8849427, 12 pages, 2020. 